\title{
Bioorthogonal photocatalytic decaging-enabled spatiotemporal proteomics
}

Zongyu Huang ${ }^{1 \#}$, Ziqi Liu ${ }^{1 \#}$, Xiao Xie ${ }^{1}$, Ruxin Zeng ${ }^{1}$, Zujie Chen ${ }^{1}$, Linghao Kong ${ }^{2}$, Xinyuan Fan ${ }^{1 *}$, Peng R. Chen ${ }^{12^{*}}$

${ }^{1}$ Synthetic and Functional Biomolecules Center, Key Laboratory of Bioorganic Chemistry and Molecular Engineering of Ministry of Education, Beijing National Laboratory for Molecular Sciences, College of Chemistry and Molecular Engineering, Peking University, Beijing 100871, China.

${ }^{2}$ Peking-Tsinghua Center for Life Sciences, Peking University, Beijing 100871, China.

${ }^{\#}$ These authors contributed equally to this work.

*Correspondence: xinyuanfan@pku.edu.cn, pengchen@pku.edu.cn

\begin{abstract}
Spatiotemporally resolved dissection of subcellular proteome is crucial to our understanding of cellular functions in health and disease. Although enzyme-based proximity labeling strategies have emerged as powerful methods to portray the compartmentalized proteome of diverse organelles, current approaches still suffer from limitations such as the genetic operation that is not compatible with hard-to-transfect cells as well as overexpression of the fusion protein that may cause altered intracellular localization. We herein report anon-genetic strategy termed bioorthogonal and photocatalytic decaging-mediated proximity labeling (CAT-Prox) for spatiotemporally resolved proteome profiling in living cells. Our systematic survey of the organometallic photocatalysts has led to the identification of $\operatorname{Ir}(\text { ppy })_{2}$ bpy as a bioorthogonal and mitochondria-targeting complex that allowed photo-controlled, rapid rescue of azidobenzyl-caged quinone methide as a highly reactive Michael acceptor for proximity-based protein labelings. By coupling with quantitative mass spectrometry, CAT-Prox revealed the dynamic mitochondria proteome of cancer cells and macrophage cellsunder normal and stressed conditions. Furthermore, by targeting the photocatalyst to cell membrane receptors, CAT-Prox allowed microdomain proteome profiling on live cell surface. Together, CAT-Prox integrated the advantages of both enzymatic and chemical-based proximity labeling approaches as a general spatiotemporal proteomics platform for diverse subcellular spaces and cell types.
\end{abstract}


The spatiotemporally organized subcellular proteome (e.g. protein abundance, turnover rates, interactions, post-translational modifications) is fundamental to virtually all life processes ${ }^{1,2}$. For example, as the powerhouse of cells, the dynamic proteome in mitochondria plays crucial roles in maintaining cellular processes such as energy and redox homeostasis ${ }^{3}$. Perturbation of mitochondrial proteome homeostasis has been linked to many human diseases such as cancer, metabolic disease and neurodegenerative disorders ${ }^{4}$. As a result, dissection of the subcellular proteome of different cell types, especially in a spatiotemporally resolved manner, may facilitate the mechanistic elucidation of biological processes as well as human diseases ${ }^{5}$. Conventional approaches require isolation of organelles after cell lysis, which suffers from low temporal resolution and possible contamination with other subcellular compartments ${ }^{6}$. Enzyme-mediated proximity labeling (e.g. APEX, TurboID) have been developed for subcellular proteome enrichment via in-situ generated reactive chemical species (e.g. phenol radical or biotinylating intermediate) $)^{7,8}$. However, such methods are largely affected by the property of the fusion bait protein which may have multiple or altered subcellular locations when overexpressed inside cells ${ }^{2}$. Furthermore, the gene transfection procedures rendered these methods difficult for hard-to-transfect cells (e.g., macrophage cells), primary neuron or tissue samples. The recently reported chemical probe-based approaches coupled the subcellular-targeting group with reactive moieties for mitochondria proteome profiling in primary neuron cells (e.g. ORMs), which had potential advantages by avoiding genetic operations ${ }^{9-11}$. However, the direct usage of reactive probes might cause offlabeling given the potent reactivities when passing through the cellular membrane and cytosolic space. Alternative chemical approaches have also emerged lately, including the micro-mapping of protein network by photocatalytic generation of carbene species via Dexter energy $\operatorname{transfer}^{12}$. But the intracellular labeling of the whole or subcellular proteome are still challenging for such shortranged carbene species.

Given the urgent query of subcellular proteome dynamics in diverse living settings ${ }^{13}$, we envisioned that a general platform that integrates the advantages of both enzymatic and chemical approaches is highly desirable, which would ideally fulfill: i) in-situ generation of reactive species in mitochondria to avoid plausible off-labeling, ii) catalytic activation of reactive species in a similar fashion as the enzymatic approaches, iii) straightforward and non-genetic operations for diverse cell 
types or tissue samples that is similar to the chemical approaches, iv) capability for non-invasive and remote-control with high temporal resolution, and v) modular and programmable design with broad applicability.

We herein report a bioorthogonal photocatalytic decaging chemistry-based spatiotemporal proteomics strategy (CAT-Prox) in living cells ${ }^{14,15}$. Systematic survey of bioorthogonal photocatalysts have enabled us to identify an iridium photocatalyst for rapid decaging of paraazidobenzyl protection group (PAB), allowing photo-controlled in-situ release of highly reactive quinone methide intermediates for proteome profiling. We applied this CAT-Prox strategy for dynamic dissection of mitochondria proteome of macrophage cells upon lipopolysaccharide (LPS)stimulated inflammation as well as microdomain proteome profiling on live cell surface (Fig. 1a).

We started by developing the bioorthogonal decaging chemistry with major considerations on modifiable catalysts for spatial targeting to mitochondria, external visible light for temporal regulation and fast reaction kinetics for precise temporal control. The transformation of aryl azide to aniline was chosen as the model reaction due to its well-documented bioorthogonality, but further development of more suitable catalytic system is needed for expanding the utility of this reaction in living systems ${ }^{16-18}$. Therefore, 4-azidobenzoic acid (1) was subjected to a collection of photocatalysts $(10 \mathrm{~mol} \%)$, including ruthenium complexes, organic photosensitizers and iridium complexes, under white LED irradiation in $\mathrm{H}_{2} \mathrm{O} / \mathrm{DMSO}(1 / 1)$ with $\mathrm{NADH}$ as additive (Fig. 1b, Supplementary Table 1). The reaction was monitored using HPLC in $30 \mathrm{~min}$. While low to moderate conversions were observed with the commonly used ruthenium complexes and organic photosensitizers, the iridium complexes showed very high catalytic activities ${ }^{19-21}$. In particular, full conversion was obtained with $\left[\operatorname{Ir}\left(2^{\prime}, 4^{\prime}-\mathrm{F}_{2}-5-\mathrm{CF}_{3}-\mathrm{ppy}\right)_{2}(\mathrm{bpy})\right] \mathrm{PF}_{6}(\operatorname{Ir} 7)$. Next, the catalyst analogue Ir8 with $t \mathrm{Bu}$ substituents on the $\mathrm{C} 4$ of bipyridine ligand was tested. Full conversion was observed as well, indicating the negligible effect of alkyl group introduction, which ensured the subsequent catalyst conjugation from $\mathrm{C} 4$ for spatial targeting (Fig. 1b). To further improve the reaction rate, blue LED light $\left(\lambda_{\max }=450 \mathrm{~nm}, 4 \mathrm{~mW} / \mathrm{cm}^{2}\right)$ was tested and the reaction was monitored using HPLC (Supplementary Fig. 1-3). Consumption of $\mathbf{1}$ along with the appearance of $\mathbf{2}$ was rapidly observed, with the conversion completed within 15 min. In addition, it was noted that the persistence of Ir8 in the HPLC traces indicated the sustainability of the catalyst for the reaction, further facilitating its 
utility as enzyme mimic in living systems (Supplementary Fig. 3). The cytotoxicity of Ir8 was evaluated next. Delightfully, no cytotoxicity was observed up to $2.0 \mu \mathrm{M}$ of Ir8 under reaction conditions (Supplementary Fig. 4), whereas less than $0.2 \mu \mathrm{M}$ of the catalyst was sufficiently enough in the following applications inside cells, which was far below from the safe concentration, guaranteeing the biocompatibility of the decaging reaction in living cells.

With the efficient photocatalyzed bioorthogonal decaging reaction in hand, we next aimed to design controllable reactive probes for proximity protein labeling. Although liberating highly reactive intermediates by decaging chemistry is highly challenging, we reasoned that quinone methide $^{22-24}$ is a very unique species that can be caged by masking its phenolic group until photocatalytic decaging-triggered rescue of the highly reactive Michael acceptor for covalent labeling with protein side-chains. To this end, we designed and synthesized a PAB-caged quinone methide probe (PAB-QM-Bio) tethered with a biotin handle (Fig. 1c), which could be transformed into reactive quinone methide intermediate in response to our photocatalytic decaging reaction to label neighbor proteins. The decaging efficiency was first tested in aqueous solution. Complete conversion of PAB-QM-Bio into 2-hydroxybenzaldehyde species, the product of cascade hydrolysis of the decaged quinone methide intermediate, was obtained within $15 \mathrm{~min}$ in the presence of both Ir8 (10 mol\%) and blue LED irradiation (Supplementary Fig. 5a). The labeling efficiency of the PAB-QM-Bio probe was next evaluated using bovine serum albumin (BSA) (Fig. 1c). Increased labeling intensity was observed along the increasing of Ir8 concentration, underlying the spatial specificity of protein labeling from the catalyst inside cells (Fig. 1d). Pulse-chase labeling of proteins was verified by switching of photo stimuli, revealing the temporal specificity from the external light (Fig. 1e and Supplementary Fig. 5b). Together, a bioorthogonal photocatalytic decaging-enabled proximity labeling system has been developed by integrating the unique advantages of photocatalytic chemistry and the high reactivity of decaging-triggerable quinone methide species.

We next aimed to target our photocatalyst into mitochondria (Fig. 2a). Serendipitously, we discovered that Ir8 itself was cell-membrane permeable and can gather at specific locations inside cells. Detailed analysis using confocal microscopy imaging with colocalization of MitoTracker ${ }^{\mathrm{TM}}$ Deep Red revealed that Ir8 was localized in mitochondria of all the cell lines we tested, most likely 
due to its positive charge and hydrophobic property (Fig. 2b and Supplementary Fig. 6). The Pearson's R value was determined to be 0.76 and 0.83 in HeLa and RAW264.7 cells respectively, indicating the efficient targeting specificity of Ir8 to mitochondria (Fig. 2c). To verify the catalytic activity of Ir8 in mitochondria, we synthesized PAB-caged rhodamine as the fluorogenic reporter, with the decaging-triggered conversion of PAB-rhodamine to rhodamine monitored by the rescued fluorescence (Fig. 2d). We incubated HeLa cells with Ir8 (200 nM) and MitoTracker ${ }^{\mathrm{TM}}$ Deep Red $(100 \mathrm{nM})$ in culture media for $30 \mathrm{~min}$. After washing, cells were charged with the PAB-rhodamine probe $(50 \mu \mathrm{M})$ and irradiated under blue LED for $15 \mathrm{~min}$. By confocal microscopy, we observed significant fluorescence of rescued rhodamine that colocalized with MitoTracker ${ }^{\mathrm{TM}}$ Deep Red, indicating the maintained catalytic activity of Ir8 in mitochondria in living cells (Fig. 2e, 2f).

With all the essential elements of CAT-Prox ready in hand, we next examined mitochondrial protein labeling in living cells (Fig. 2g). Live HeLa cells were subjected to CAT-Prox protocol, and immunofluorescence analysis was conducted subsequently. The co-localization of biotin immunofluorescence with mitochondrial marker TOMM20 indicated the in-situ labeling of proteins in mitochondria by CAT-Prox (Fig. 2h). The protein labeling efficiency was evaluated by immunoblotting next. The mitochondria in live cells were charged with Ir8 (ranging from 0 to 0.2 $\mu \mathrm{M})$, followed by PAB-QM-Bio probe addition $(100 \mu \mathrm{M})$ and blue LED irradiation up to10 min. After cell lysis, the biotinylated proteome was subjected to immunoblotting analysis which showed sufficient protein labeling with $0.2 \mu \mathrm{M}$ catalyst loading and $10 \mathrm{~min}$ photo irradiation (Fig. 2i).

By combining with the protein mass spectrometry, CAT-Prox would offer a powerful tool for mitochondria proteome profiling in living cells, which may facilitate the understanding of carcinogenic mechanism in cancer cells. To verify our method, we applied CAT-Prox to HeLa cells and the biotin labeled proteins were enriched with streptavidin-coated beads and analyzed by LCMS/MS (Supplementary Fig. 7). Three independent experiments revealed 258 proteins with 182 mitochondria proteins identified according to MitoCarta3.0 database (70.5\%) (Fig 3a). Submitochondrial analysis of the identified proteins revealed that the captured proteome maintained the original distribution profile (Fig 3a, Supplementary Fig. 8). Moreover, with Gene Ontology (Biological Process) analysis powered by Metascape ${ }^{25}$, we noticed that the citric acid cycle (TCA)related proteins were highly enriched from HeLa cells, consistent with the increased metabolic 
activities of cancer cells (Fig. 3b) ${ }^{26}$.

The mitochondrial proteome of immune cells can undergo highly dynamic regulation during immunological processes, including innate immune signaling as well as adaptive immune activation $^{27}$. It is often challenging to dissect proteome in immune cells by using enzymatic methods due to the natural defense to gene transfection of such cells ${ }^{28}$. We were interested in applying our CAT-Prox mitochondrial proteome profiling in macrophage cells by using RAW264.7 cells as the model. A total of 300 proteins were detected from three independent CAT-Prox experiments, with 216 mitochondrial proteins identified (72\% specificity), demonstrating the high efficiency of CATProx for mitochondrial proteome profiling in macrophage cells (Fig. 3c). Gene Ontology analysis revealed that proteins related with mitochondrial transport were particularly detected in RAW264.7 macrophage cells, likely due to the rapid transportation of metabolites (e.g. citrate, glutamine) that are essential for immunity maintenance (Fig. 3d) ${ }^{27}$. The proteome difference between HeLa and RAW264.7 cells reflected the correlation between mitochondrial proteomes and cell functions.

Since macrophage inflammatory responses play crucial roles during processes such as bacterial infection and inflammatory diseases ${ }^{29}$, we hope to examine the mitochondria proteome dynamics upon inflammatory response of macrophages. CAT-Prox was conducted with the lipopolysaccharide (LPS)-stimulated RAW264.7 cells, and the enriched proteins were analyzed by quantitative mass spectrometry with normal RAW264.7 cells as a control (Fig. 3e). Upregulation (e.g. IRG1, Aco2, Sod2, Acadsb and Dld) and downregulation (e.g. Acot13, Acp6) of mitochondrial proteins were detected in inflammatory response (Fig. 3f, Supplementary Fig. 9). We chose the top three upregulated proteins (IRG1, Aco2 and Sod2) for further immunoblotting analysis. IRG1 found as the most upregulated protein detected by both CAT-Prox and immunoblotting, indicating the high fidelity of CAT-Prox-based proteomics (Fig. 3g). Noteworthy, Aco2 (aconitase 2) and IRG1 (aconitate decarboxylase 1) were responsible for itaconate production in a tandem manner ${ }^{30}$, agreeing with the previous discovery of enhanced itaconate production in LPS-stimulated macrophages ${ }^{31}$. Similarly, the increase of reactive electrophile species (RES) ${ }^{32}$ was likely due to the upregulation of $\mathrm{Acadsb}^{33}$. The upregulation of the superoxide anion radical scavenger superoxide dismutase [Mn] (Sod2) was probably a self-defense response to the LPS-induced oxidative stress ${ }^{34}$.

To further demonstrate the generality of our CAT-Prox strategy, we further targeted the Ir8 
photocatalysis to cell surface for the proteome profiling of microdomains on cell membrane, which often plays important roles in cellular functions, such as membrane organization ${ }^{35}$ and signal transductions ${ }^{36}$. Thus, antibody-catalyst conjugate $(\operatorname{Her}[\operatorname{Ir}])$ was designed and prepared via the CuAAC ligation between the alkynyl-modified photocatalyst $\operatorname{Ir} 9$ and azide-functionalized Herceptin, for targeting the catalyst to the tumor cell surface by affinity binding of Herceptin to its receptor HER2 (Supplementary Fig. 10). Upon Her[Ir] targeting to live HER2 ${ }^{+}$MDA cell surface, CAT-Prox labeling was performed. Efficient and selective protein labeling was verified by subsequent immunoblotting, flowcytometry, and immunofluorescent imaging analysis respectively (Supplementary Fig. 11). The labeled proteins were further analyzed by protein mass spectrometry (Supplementary Fig. 12). Besides HER2 itself as well as subunits of Herceptin (IGKC and IGHG1), additional 7 proteins were significantly enriched (Supplementary Fig. 12c). For example, detection of GLUT1 (SLC2A1), a glucose transporter previously reported to be required in HER2-induced tumorigenesis ${ }^{37}$, suggested the correlation between its function and spatial proximity to HER2. STRING network analysis ${ }^{38}$ and RaftProt (V2) database search $^{39}$ indicated that the network was significantly enriched with PPIs $(p<0.01)$, suggesting a possible network of these proteins in a microdomain on cell membrane (Supplementary Fig. 12d-f) ${ }^{40}$.

In summary, we have developed a photocatalytic proteome profiling method (CAT-Prox) with facile spatiotemporal controllability by integrating the unique advantages of photocatalytic decaging chemistry and the protein-reactive quinone methide species, allowing dynamic dissection of subcellular proteome of hard-to-transfect cells. The validation of CAT-Prox is exampled by proteome profiling of mitochondria and surface microdomain of cancer cells or macrophage cells. By combining CAT-Prox with quantitative mass spectrometry, we achieved dynamic mitochondrial proteome profiling upon macrophage inflammation response, which revealed the underlying causes for metabolite change and self-defense during inflammatory processes. CAT-Prox was also performed on cell surface for microdomain proteome profiling by surface-targeting of the photocatalyst, demonstrating the generality of our CAT-Prox strategy to various intracellular and cell surface spaces. The advantages of our approach include the catalytic and in-situ generation of the reactive labeling probe, avoiding of issues from genetic operation and bait protein, remotecontrollability by external photo stimuli, as well as modular design from the decaging pairs, which 
enabled CAT-Prox as a general platform for dynamic subcellular proteome profiling in living cells. Expansions of CAT-Prox are straightforward in multiple directions, such as extending to other subcellular organelles by targeting photocatalysts to the desired space, imaging-guided proteomics dissection by taking advantage of the fluorescent property of the photocatalyst, as well as applications in broad biological dynamic processes.

\section{Methods}

Materials, protocols and data characterizations for all biological and chemical experiments are described in detail in the Supplementary Information.

\section{Acknowledgements}

We acknowledge funding from the Ministry of Science and Technology (2019YFA0904201, 2016YFA0501500), the National Natural Science Foundation of China (91957101, 22077004, 21740001, 21937001), and Beijing Natural Science Foundation (Z200010).

\section{Author contributions}

X.F. and P.C. conceived and supervised the project. Z. H., Z. L., X. X., R. Z. and Z. C. performed the experiments. Z.L., Z.H., and L.K. analyzed the data. X.F., P.C., Z.L. and Z.H. wrote the paper with contributions from all the authors.

\section{Competing interests}

The authors declare no competing interests.

\section{References}

1. Thul, P.J. et al. A subcellular map of the human proteome. Science 356, eaal3321 (2017).

2. Christopher, J.A. et al. Subcellular proteomics. Nat. Rev. Met. Primers 1, 32 (2021).

3. Nunnari, J. \& Suomalainen, A. Mitochondria: in sickness and in health. Cell 148, 1145-1159 (2012).

4. Song, J., Herrmann, J.M. \& Becker, T. Quality control of the mitochondrial proteome. Nat. Rev. Mol. Cell Biol. 22, 54-70 (2021).

5. Singh, A. Subcellular proteome map of human cells. Nat. Methods 18, 713-713 (2021).

6. Frezza, C., Cipolat, S. \& Scorrano, L. Organelle isolation: functional mitochondria from mouse liver, muscle and cultured filroblasts. Nat. Protoc. 2, 287-295 (2007).

7. Branon, T.C. et al. Efficient proximity labeling in living cells and organisms with TurboID. Nat. Biotechnol. 36, 880-887 (2018).

8. Rhee, H.W. et al. Proteomic mapping of mitochondria in living cells via spatially restricted enzymatic tagging. Science 339, 1328-1331 (2013).

9. Yasueda, Y. et al. A Set of Organelle-Localizable Reactive Molecules for Mitochondrial Chemical Proteomics in Living Cells and Brain Tissues. J. Am. Chem. Soc. 138, 7592-7602 (2016).

10. Fujisawa, A., Tamura, T., Yasueda, Y., Kuwata, K. \& Hamachi, I. Chemical Profiling of the Endoplasmic Reticulum Proteome Using Designer Labeling Reagents. J. Am. Chem. Soc. 140, 17060-17070 (2018). 
11. Shiraiwa, K., Cheng, R., Nonaka, H., Tamura, T. \& Hamachi, I. Chemical Tools for Endogenous Protein Labeling and Profiling. Cell Chem. Biol. 27, 970-985 (2020).

12. Geri, J.B. et al. Microenvironment mapping via Dexter energy transfer on immune cells. Science 367, 1091 (2020).

13. Song, J., Herrmann, J.M. \& Becker, T. Quality control of the mitochondrial proteome. Nat. Rev. Mol. Cell Biol. 22, 54-70 (2021)

14. Li, J. \& Chen, P.R. Development and application of bond cleavage reactions in bioorthogonal chemistry. Nat. Chem. Biol. 12, 129-137 (2016).

15. Wang, J., Wang, X., Fan, X. \& Chen, P.R. Unleashing the Power of Bond Cleavage Chemistry in Living Systems. ACS Cent. Sci. 7, 929-943 (2021).

16. Chen, Y., Kamlet, A.S., Steinman, J.B. \& Liu, D.R. A biomolecule-compatible visible-light-induced azide reduction from a DNA-encoded reaction-discovery system. Nat. Chem. 3, 146-153 (2011).

17. Sadhu, K.K., Eierhoff, T., Romer, W. \& Winssinger, N. Photoreductive uncaging of fluorophore in response to protein oligomers by templated reaction in vitro and in cellulo. J. Am. Chem. Soc. 134, 20013-20016 (2012).

18. Angerani, S. \& Winssinger, N. Visible Light Photoredox Catalysis Using Ruthenium Complexes in Chemical Biology. Chem. Eur. J. 25, 6661-6672 (2019).

19. Prier, C.K., Rankic, D.A. \& MacMillan, D.W. Visible light photoredox catalysis with transition metal complexes: applications in organic synthesis. Chem. Rev. 113, 5322-5363 (2013).

20. Fan, X., Gong, X., Ma, M., Wang, R. \& Walsh, P.J. Visible light-promoted CO2 fixation with imines to synthesize diaryl alpha-amino acids. Nat. Commun. 9, 4936 (2018).

21. Zhu, J., Dai, C., Ma, M., Yue, Y. \& Fan, X. Visible light-mediated cross-coupling of electrophiles: synthesis of $\alpha$-amino amides from isocyanates and ketimines. Org. Chem. Front. 8, 1227-1232 (2021).

22. Weinert, E.E. et al. Substituents on Quinone Methides Strongly Modulate Formation and Stability of Their Nucleophilic Adducts. J. Am. Chem. Soc. 128, 11940-11947 (2006).

23. Liu, J. et al. Photocaged Quinone Methide Crosslinkers for Light-Controlled Chemical Crosslinking of ProteinProtein and Protein-DNA Complexes. Angew. Chem. Int. Ed. 58, 18839-18843 (2019).

24. Liu, J. et al. Genetically Encoded Quinone Methides Enabling Rapid, Site-Specific, and Photocontrolled Protein Modification with Amine Reagents. J. Am. Chem. Soc. 142, 17057-17068 (2020).

25. Zhou, Y. et al. Metascape provides a biologist-oriented resource for the analysis of systems-level datasets. Nat. Commun. 10, 1523 (2019).

26. Anderson, N.M., Mucka, P., Kern, J.G. \& Feng, H. The emerging role and targetability of the TCA cycle in cancer metabolism. Protein Cell 9, 216-237 (2018).

27. Mills, E.L., Kelly, B. \& O'Neill, L.A.J. Mitochondria are the powerhouses of immunity. Nat. Immunol. 18, 488498 (2017)

28. Jiang, W., Reich, I.C. \& Pisetsky, D.S. Mechanisms of activation of the RAW264.7 macrophage cell line by transfected mammalian DNA. Cell. Immunol. 229, 31-40 (2004).

29. Medzhitov, R. Origin and physiological roles of inflammation. Nature 454, 428-435 (2008).

30. Wang, Y., Li, N., Zhang, X. \& Horng, T. Mitochondrial metabolism regulates macrophage biology. J. Biol. Chem. 297, 100904 (2021).

31. Lampropoulou, V. et al. Itaconate Links Inhibition of Succinate Dehydrogenase with Macrophage Metabolic Remodeling and Regulation of Inflammation. Cell Metab. 24, 158-166 (2016).

32. Timblin, G.A. et al. Mitohormesis reprogrammes macrophage metabolism to enforce tolerance. Nat. Metab. 3 , 618-635 (2021).

33. Farmer, E.E. \& Davoine, C. Reactive electrophile species. Curr. Opin. Plant Biol. 10, 380-386 (2007). 
34. West, A.P. et al. TLR signalling augments macrophage bactericidal activity through mitochondrial ROS. Nature 472, 476-480 (2011).

35. Lingwood, D. \& Simons, K. Lipid rafts as a membrane-organizing principle. Science 327, 46-50 (2010).

36. Harder, T. Lipid raft domains and protein networks in T-cell receptor signal transduction. Curr. Opin. Immunol. 16, 353-359 (2004).

37. Wellberg, E.A. et al. The glucose transporter GLUT1 is required for ErbB2-induced mammary tumorigenesis. Breast Cancer Res. 18, 131 (2016).

38. Szklarczyk, D. et al. STRING v11: protein-protein association networks with increased coverage, supporting functional discovery in genome-wide experimental datasets. Nucleic Acids Res. 47, D607-D613 (2019).

39. Mohamed, A., Shah, A.D., Chen, D. \& Hill, M.M. RaftProt V2: understanding membrane microdomain function through lipid raft proteomes. Nucleic Acids Res. 47, D459-D463 (2019).

40. Shah, A.D. et al. Integrative Analysis of Subcellular Quantitative Proteomics Studies Reveals Functional Cytoskeleton Membrane-Lipid Raft Interactions in Cancer. J. Proteome Res. 15, 3451-3462 (2016). 


\section{Figures}

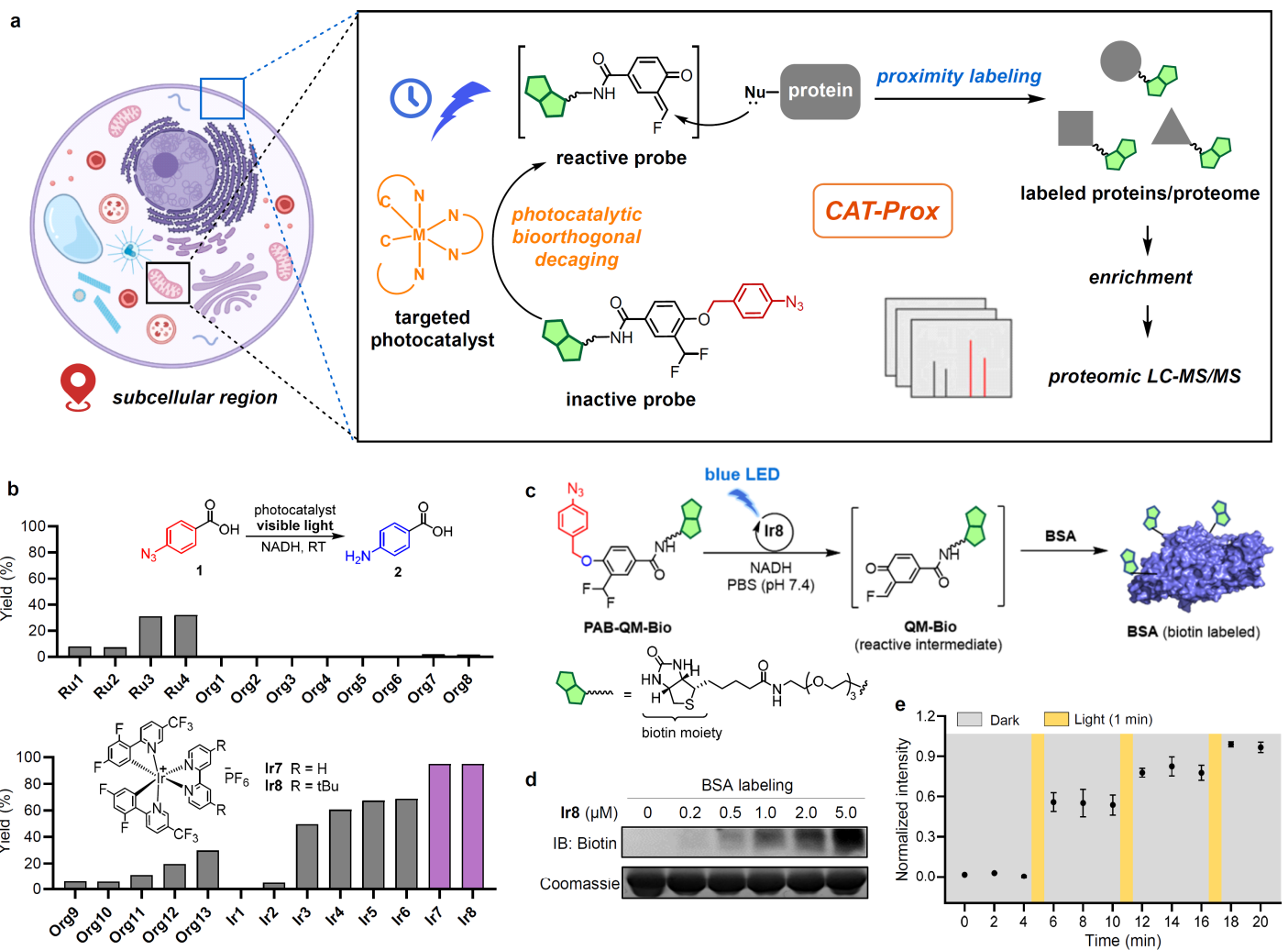

Fig.1 Development of the bioorthogonal and photocatalytic decaging-enabled proximity labeling strategy (CAT-Prox). a, Schematic illustration of the CAT-Prox. b, Catalyst screening for the aryl azide reduction reaction. The reaction was carried out with $\mathbf{1}(0.1 \mathrm{mmol})$ and NADH $(0.2$ mmol) in $0.5 \mathrm{~mL}$ mixture of water and DMSO (1/1) with photocatalyst (10 mol\%, $0.01 \mathrm{mmol})$ at room temperature under white LED irradiation for $30 \mathrm{~min}$. c, Bioorthogonal decaging-enabled proximity labeling of proteins via rescuing of the reactive molecule — quinone methide — based on the photocatalyzed decaging chemistry. d, Photocatalyst concentration-dependent labeling of BSA with PAB-QM-Bio probe in vitro. Biotinylated BSA and total BSA were analyzed by immunoblotting and Coomassie blue staining, respectively. e, Time-resolved BSA labeling by switching of the photo stimuli. Quantification of biotinylating level was based on immunoblotting analysis (normalized blot intensity against total protein stain, $n=3$ ). 

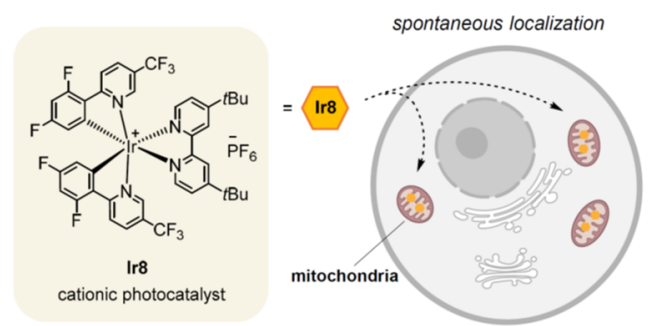
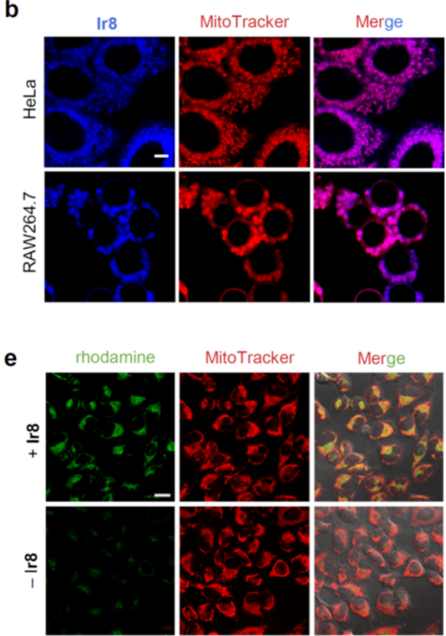

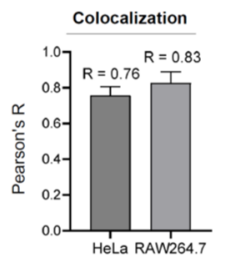

f

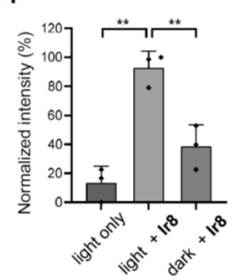

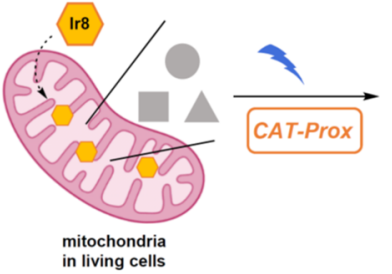
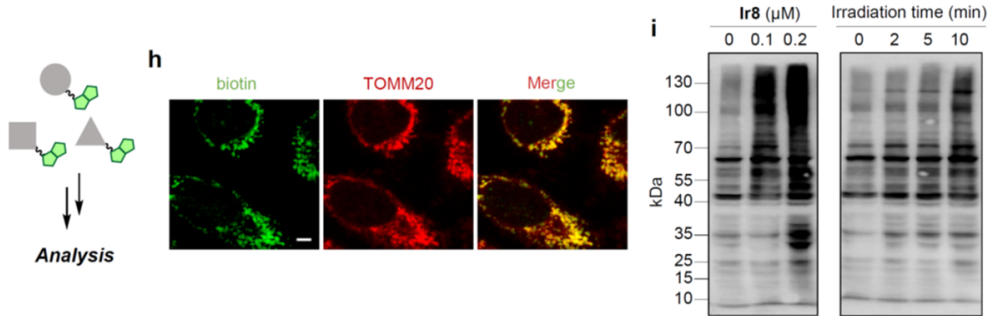

Fig.2 Development of CAT-Prox as a non-genetic, enzyme-like strategy for mitochondria proteomics. a, Targeting of Ir8 photocatalyst into mitochondria via self-localization. b, Confocal microscopic images of cells treated with Ir8 ( $1 \mu \mathrm{M}$, blue) and MitoTracker ${ }^{\mathrm{TM}}$ Deep Red (100 nM, red) indicated the mitochondria-targeting of Ir8 itself. Scale bar, $5 \mu \mathrm{m}$. c, Pearson's R value for evaluation of the colocalization in living cells. $R=0.76$ and 0.83 for HeLa cells $(n=21)$ and RAW264.7 cells $(n=25)$, respectively. $\mathbf{d}$, Photo-triggered fluorescence rescue by PAB-rhodamine decaging in mitochondria. e, Confocal microscopic images showing the fluorescence of the rescued rhodamine 110 in the mitochondria of HeLa cells pre-treated with Ir8 (200 nM) and irradiated by blue LED (15 min). Samples without Ir8 treatment were used as control. Scale bar, $20 \mu \mathrm{m}$. f, Fluorescent quantification of the rescued rhodamine 110 in HeLa cells by flowcytometry. Data are represented as mean \pm s.d. $\left(\mathrm{n}=3\right.$ independent samples). ${ }^{* *} p<0.0021$, unpaired two-tailed $t$-test. $\mathbf{g}$, Photo-controlled chemical proteomic profiling in mitochondria. $\mathbf{h}$, Immunofluorescence analysis of the HeLa cells after CAT-Prox labeling verified the in-situ protein labeling in mitochondria. Scale bar, $5 \mu \mathrm{m}$. i, Immunoblotting analysis of biotinylated proteins after CAT-Prox treatment of HeLa cells with Ir8 (0-200 nM), PAB-QM-biotin probe (100 $\mu \mathrm{M})$ and blue LED irradiation (0-10 min). 

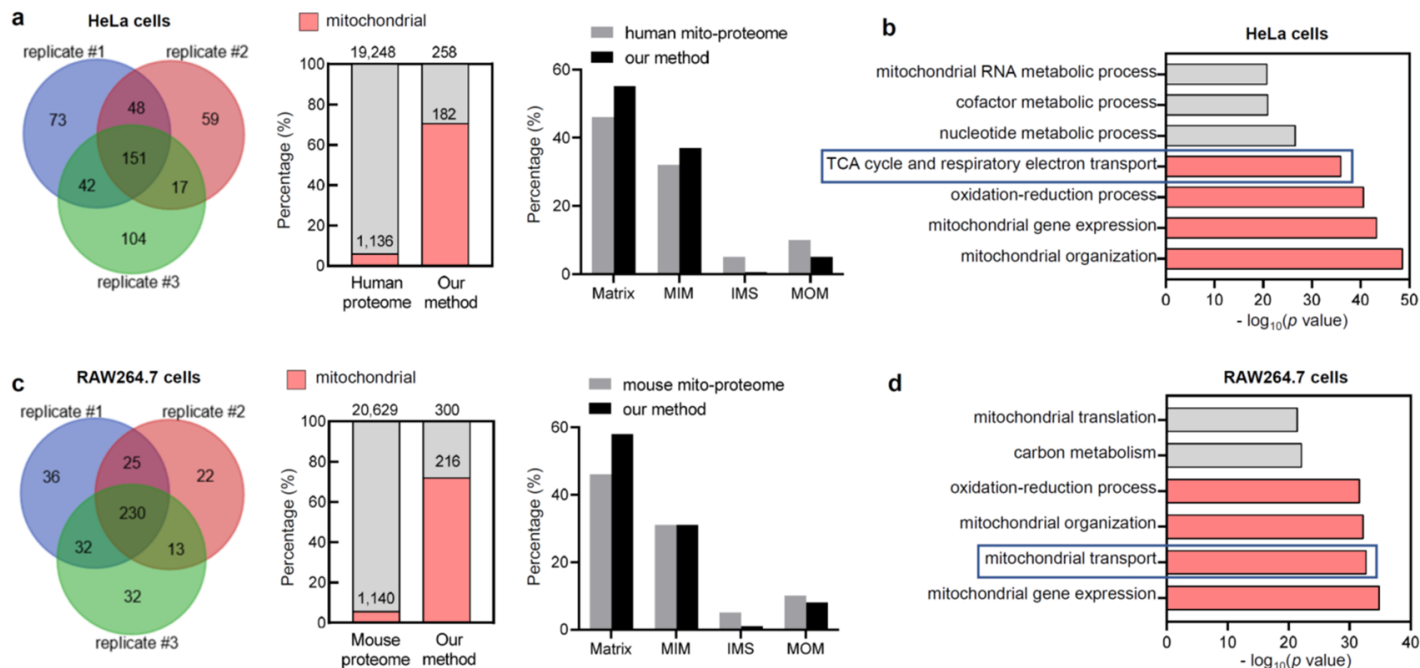

d
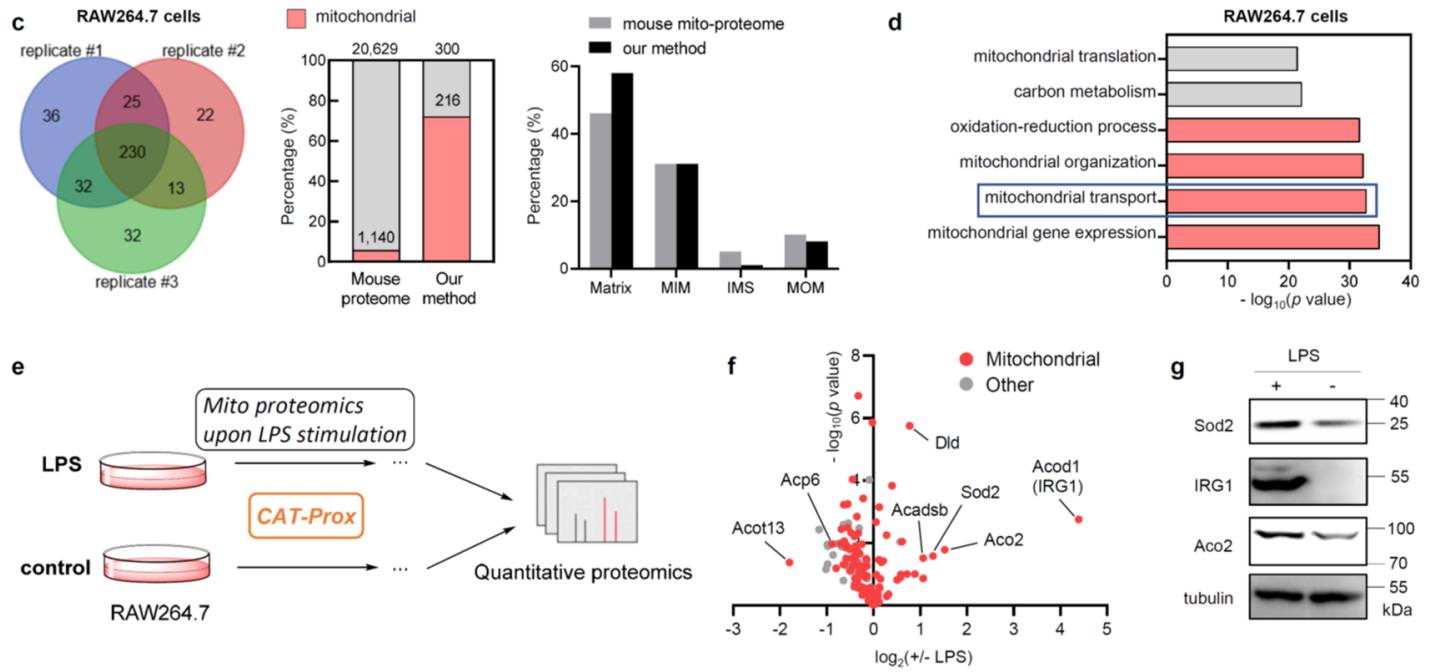

IFig.3 CAT-Prox-enabled mitochondria proteome profiling and dynamics in living cells. a,

Combination of CAT-Prox with protein mass spectrometry to identify the labeled proteins in HeLa cells. 182 mitochondrial proteins were identified from 258 enriched proteins according to the MitoCarta3.0 database (70.5\% specificity). Proteins identified as mitochondrial proteins were classified into 'matrix', 'mitochondrial inner membrane (MIM)', 'intermembrane space (IMS)' and 'mithochondrial outer membrane (MOM)'. b, Gene Ontology (GO) analysis (Biological Processes) for identified protein from HeLa cells. c, Protein identification in RAW264.7 cells. 216 mitochondrial proteins from 300 enriched proteins were identified ( $72 \%$ specificity). d, GO analysis of the identified proteins from RAW264.7 cells. e, Combination of CAT-Prox with quantitative protein mass spectrometry to dissect the mitochondria proteome dynamics during the inflammatory process of LPS stimulated-RAW264.7 cells. f, Volcano blot showing the regulation of mitochondrial proteins in the inflammatory process of RAW264.7 cells. g, Immunoblotting analysis of the representative proteins identified above. 